\title{
Comparison of repeated transcranial stimulation and transcranial direct-current stimulation on primary motor cortex excitability and inhibition: A pilot study
}

\author{
Vincent Cabibel ${ }^{1,2, *}$, Makii Muthalib ${ }^{1,4,5}$, Jérôme Froger ${ }^{1,3}$, and Stéphane Perrey ${ }^{1}$ \\ 1 EuroMov, Univ. Montpellier, 700 Avenue du Pic St Loup, Montpellier 34090, France \\ ${ }^{2}$ Clinique du Souffle les Clarines, Riom-Es-Montagne 15400, France \\ 3 Department MPR, CHU Nîmes, Grau du Roi 30240, France \\ 4 School of Psychology, Deakin University, Melbourne 3125, Australia \\ ${ }^{5}$ Silverline Research Services, Brisbane 4127, Australia
}

Accepted 11 December 2017

\begin{abstract}
Repeated transcranial magnetic stimulation (rTMS) is a well-known clinical neuromodulation technique, but transcranial direct-current stimulation (tDCS) is rapidly growing interest for neurorehabilitation applications. Both methods (contralesional hemisphere inhibitory low-frequency: LF-rTMS or lesional hemisphere excitatory anodal: a-tDCS) have been employed to modify the interhemispheric imbalance following stroke. The aim of this pilot study was to compare aHD-tDCS (anodal high-definition tDCS) of the left M1 (2 mA, $20 \mathrm{~min})$ and LF-rTMS of the right M1 (1 Hz, $20 \mathrm{~min})$ to enhance excitability and reduce inhibition of the left primary motor cortex (M1) in five healthy subjects. Single-pulse TMS was used to elicit resting and active (low level muscle contraction, $5 \%$ of maximal electromyographic signal) motor-evoked potentials (MEPs) and cortical silent periods (CSPs) from the right and left extensor carpi radialis muscles at Baseline, immediately and $20 \mathrm{~min}$ (Post-Stim-20) after the end of each stimulation protocol. LF-rTMS or aHD-tDCS significantly increased right M1 resting and active MEP amplitude at Post-Stim-20 without any CSP modulation and with no difference between methods. In conclusion, this pilot study reported unexpected M1 excitability changes, which most likely stems from variability, which is a major concern in the field to consider.
\end{abstract}

Keywords: non-invasive brain stimulation, contralateral hemisphere, interhemispheric interactions

Résumé- Comparaison entre les effets de la stimulation magnétique transcrânienne répétée et la stimulation transcrânienne à courant continu sur l'excitabilité du cortex moteur primaire: une étude pilote. En neuro-réhabilitation, la stimulation magnétique transcrânienne répétée (rTMS) est reconnue alors que la stimulation transcrânienne à courant continu (tDCS) tend à fortement se développer. Chacune des méthodes (inhibition controlatérale via rTMS basse fréquence, LF-rTMS, ou renforcement de l'hémisphère lésé via tDCS anodale, a-tDCS) est utilisée pour rétablir le déséquilibre interhémisphérique suite à un accident vasculaire cérébral. Le but de cette étude pilote était de comparer les effets induits par aHD-tDCS (tDCS anodale haute-définition, $20 \mathrm{~min}, 2 \mathrm{~mA})$ du cortex moteur primaire (M1) gauche et LF-rTMS $(20 \mathrm{~min}, 1 \mathrm{~Hz})$ de M1 droit pour augmenter l'excitabilité et diminuer l'activité inhibitrice de M1 gauche chez cinq sujets sains adultes. Les potentiels moteurs évoqués (MEP) par TMS au repos et lors de faibles contractions (actif, $5 \%$ de l'activité électromyographique maximale) associés aux périodes de silences (CSP) étaient recueillis au niveau des muscles extenseurs radial du carpe droit et gauche avant (Baseline), immédiatement après et 20 min après (Post-Stim-20) chaque méthode. Aucune variation des CSP n'a été observée pour les deux méthodes mais l'amplitude des MEP de repos et actif de M1 droit était significativement plus importante à Post-Stim-20. Cette étude pilote rapporte des modulations d'excitabilité majoritairement attribuables à la variabilité des réponses interindividuelles.

Mots clés : interactions interhémisphériques, hémisphère controlatéral, stimulation corticale non invasive

\footnotetext{
*Corresponding author: vincent.cabibel@umontpellier.fr
} 


\section{Introduction}

Experimental interventions that might alleviate poststroke motor impairments include non-invasive brain stimulation (NIBS) in order to inhibit activity in the contralesional primary motor cortex (M1) and facilitate activity in the ipsilesional M1. Although repeated transcranial magnetic stimulation (rTMS) is a well-known clinical neuromodulation technique (Corti, Patten, \& Triggs, 2012; Hoyer \& Celnik, 2011; Schulz, Gerloff, \& Hummel, 2013), transcranial direct-current stimulation (tDCS) is rapidly growing interest as a more simple and affordable method. Based on the interhemispheric inhibition principle, stating a mutual inhibition between hemispheres (Ferbert et al., 1992), low frequency rTMS (LF-rTMS) is applied to the contralesional hemisphere (Corti et al., 2012; Schambra, Sawaki, \& Cohen, 2003); while anodal tDCS (a-tDCS) is applied to the affected/ ipsilesional hemisphere (Schlaug, Renga, \& Nair, 2008). Both NIBS methods aim to counterbalance the loss in excitability of the affected hemisphere and restore the balance between hemispheres (Hao, Wang, Zeng, \& Liu, 2013; Nowak, Grefkes, Ameli, \& Fink, 2009).

Repeated transcranial magnetic stimulation (rTMS) was derived from single-pulse transcranial magnetic stimulation (TMS) developed 30 years ago (Barker, Jalinoous, \& Freeston, 1985). The magnetic stimuli are repeated over time to modulate the M1 excitability and induce neuroplasticity (Schulz et al., 2013). Since its approval as a recognized therapy for depression by the Food and Drug Administration (Wassermann, 1998), rTMS has become a gold NIBS standard in neurorehabilitation. LF-rTMS is generally known to decrease the M1 excitability when applied with a 1 to $5 \mathrm{~Hz}$ interstimulus interval at intensities ranging $\sim 70-90 \%$ of the motor threshold (Ridding \& Rothwell, 2007) and durations ranging from 0 to $30 \mathrm{~min}$ (Arai et al., 2007; Maeda, Keenan, Tormos, Topka, \& Pascual-Leone, 2000); at high frequency $(\mathrm{HF}-\mathrm{rTMS}>5 \mathrm{~Hz})$, M1 excitability is increased. Furthermore, decreasing one M1's excitability with LF-rTMS would reduce its inhibitory activity toward the opposite M1 that sees in turn its excitability increases as previously reported in healthy subjects (Gilio, Rizzo, Siebner, \& Rothwell, 2003). It is important to note that LF-rTMS is preferred to HF-rTMS due to its propensity to trigger seizures (Dobek, Blumberger, Downar, Daskalakis, \& Vila-Rodriguez, 2015; Pereira, Muller, da Mota Gomes, Rotenberg, \& Fregni, 2016).

The tDCS research field is growing interest as a promising neurorehabilitation technique (Chang, Kim, \& Park, 2015; Di Lazzaro et al., 2014; Jo et al., 2009; Simonetta-Moreau, 2014). With a conventional anodal tDCS (a-tDCS) montage, a large rubber-sponge anode electrode is applied onto the scalp overlying the M1 region of interest and the cathode as the reference electrode is placed on the contralateral supra orbital area. In healthy subjects, this montage has usually shown to increase M1 excitability as determined by TMS motor-evoked potentials (MEP) amplitude (Gandiga, Hummel, \& Cohen, 2006; Nitsche \&
Paulus, 2000). For example, an increase in M1 MEP amplitude up to $35 \%$ above baseline has been documented after $2 \mathrm{~mA}$ anodal tDCS of the left M1 (Jeffery, Norton, Roy, \& Gorassini, 2007). Recently, advances in the research field led to the development of high-definition (HD)-tDCS to constrain the current in the area of interest and increase its efficiency (Edwards et al., 2013; Kuo et al., 2013; Muthalib, Besson, Rothwell, Ward, \& Perrey, 2016b; Villamar et al., 2013). This particular montage uses an active electrode (anode, in the case of anodal stimulation) surrounded by four return reduced size electrodes $35 \mathrm{~mm}$ away instead of the standard bi-electrode montage.

To the best of our knowledge, only a few studies (Priori, Hallett, \& Rothwell, 2009) directly compared rTMS and tDCS techniques in a same experimental design. By comparing a-tDCS with HF-rTMS protocol, Simis et al. (2013) found an expected increased MEP amplitude following HF-rTMS but also found a surprising decreased M1 excitability with a-tDCS. They argued that this unexpected tDCS result was due to the neuronal counter-regulation principle (homeostatic metaplasticity) that prevents over-excitation of the neurons, which caused this reversal in M1 excitability (Monte-Silva et al., 2013).

The aim of this pilot study in healthy subjects was to compare anodal high-definition tDCS (aHD-tDCS) of the left M1 and LF-rTMS of the right M1 to modulate excitability and reduce inhibition (TMS evoked cortical silent period, CSP) in the left M1. Based on the literature, we hypothesized that aHD-tDCS would increase the excitability and decrease inhibition of the stimulated left M1 while LF-rTMS would decrease stimulated right M1 excitability and increase right M1 inhibition but also increase the left M1 excitability and decrease left M1 inhibition through interhemispheric inhibition principle.

Left M1 was chosen as a reference as it is the dominant hemisphere for right-handers.

\section{Materials and methods}

\subsection{Participants}

Five healthy right-handed subjects (4 males and 1 female, aged $33.2 \pm 9 \mathrm{yr}$ ) volunteered to participate in this pilot study. All participants were free from any known neuromuscular injury or neurological disorders. The experimental procedures and protocols conformed to the recommendations of the local Human Research Ethics Committee in accordance with the latest version of the Declaration of Helsinki, and all subjects gave their informed written consent prior to the experiments. The handedness was quantified using the Edinburgh inventory test (Oldfield, 1971) and presented a mean right laterality quotient of $96.4 \pm 8.1$. The experiments were run at the hospital of Grau-du-Roi (CHU Nîmes, 30240, France).

\subsection{Experimental procedure}

Figure 1 shows a schematic of the experimental design. Participants were randomly assigned to undergo aHDtDCS of the left M1 (2 mA, $20 \mathrm{~min})$ or LF-rTMS of the 


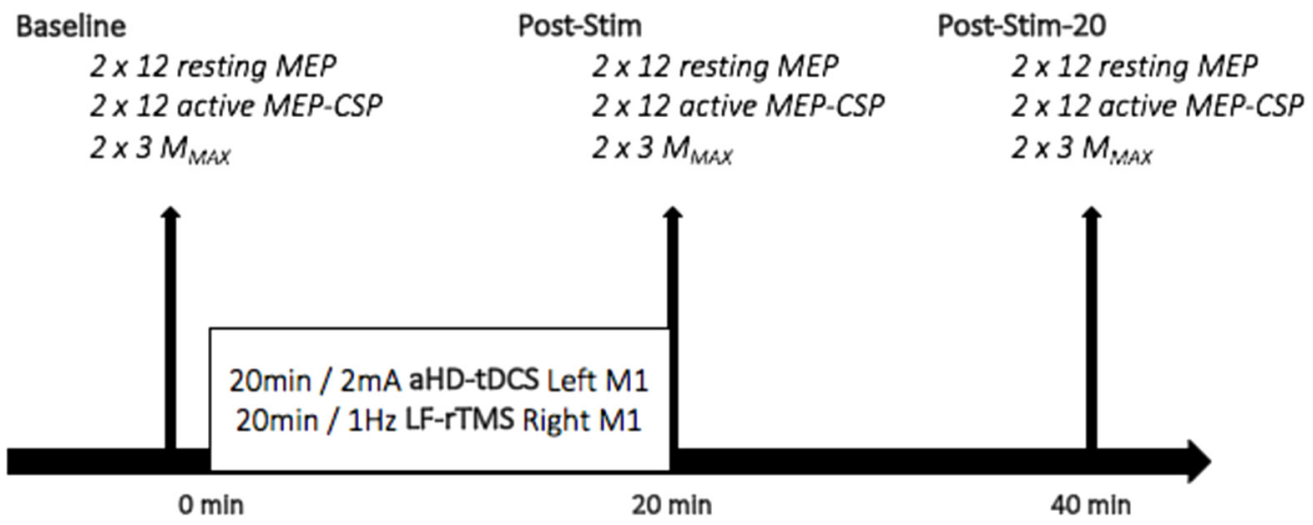

Figure 1. Schematic description of the experimental design. TMS (MEP and CSP) and nerve stimulation (MAx) measurements were performed for the left and right (as indicated by two series of measurements) M1 of each participant at Baseline, immediately (Post-Stim) and $20 \mathrm{~min}$ (Post-Stim-20) after the end of a 20-min period of neuromodulation brought by either anodal high-densitytDCS (aHD-tDCS) or low frequency-repeated TMS (LF-rTMS).

right M1 $(1 \mathrm{~Hz}, 20 \mathrm{~min})$ in a crossover design with at least 48 hrs between each session.

TMS measurements were alternatively performed from both left and right M1 in a randomised and counterbalanced order under two conditions: Resting (both arms) or Active (contralateral arm contracting in order to extent the wrist in line with the forearm to induce $\sim 5 \%$ of maximal electromyograhic - EMG - activity) (Teo et al., 2015). TMS measurements were tested at Baseline, immediately (Post-Stim) and 20 min (Post-Stim-20) after the end of the LF-rTMS or aHD-tDCS session. For each TMS measurement, 12 single-pulse MEPs were elicited from left and right M1 towards the contralateral extensor carpi radialis (ECR) muscle with a $5 \mathrm{~s}$ inter-stimulus interval; this muscle was chosen as previously used as target muscles in previous TMS studies using similar experimental design (Hendy \& Kidgell, 2014; Muthalib, Cabibel, Rothwell, Teo, \& Perrey, 2017; Teo et al., 2015). At the end of each time-point, three maximal M-waves $\left(\mathrm{M}_{\mathrm{MAX}}\right)$ were alternatively and randomly evoked from the left and right ECR muscles.

The maximal EMG activity was determined prior to the experiment for each arm during maximal voluntary contractions against a fixed support, with the best performance in three trials out of $5 \mathrm{~s}$ with a resting period of $60 \mathrm{~s}$ between each.

\subsection{Experimental setup}

Participants were sitting in a chair with the two forearms supported in a horizontal and pronated position on an armrest. The forearm and wrist were in a position such that the palm was faced down and the fingers were in line with the straight wrist. The legs were flexed and the trunk was resting on the back of the chair.

Surface EMG activity of the left and right ECR muscles was recorded with two pairs of self-adhesive silver chloride electrodes (Contrôle Grahique Medical, France) in bipolar configuration with a $20 \mathrm{~mm}$ electrode distance (Hermens, Freriks, Disselhorst-Klug, \& Rau, 2000). Low impedance $(<5 \mathrm{k} \Omega)$ between electrodes was obtained by shaving, gently abrading the skin with sandpaper and cleaning it with modified alcohol. Electrodes were positioned lengthwise over the muscle belly and the reference electrode was attached to the left wrist radial styloid process.

EMG data were recorded at a sampling frequency of $2000 \mathrm{~Hz}$ with a Biopac MP100 (Biopac Systems Inc., Santa Barbara, CA, USA) and a computer running AcqKnowledge software (Biopac Systems Inc., Santa Barbara, CA, USA). The EMG signal was amplified (500 gain) with a bandwidth frequency ranging from 10 to $500 \mathrm{~Hz}$. The root mean square of the EMG signal (rmsEMG) was calculated for the left and right ECR over 200 samples during the whole experiment. The rmsEMG was used to measure the level of EMG activity level (Li et al., 2014) during the EMG activity plateau ( $1 \mathrm{~s}$, with less than $10 \%$ of peak-topeak variation of the mean rmsEMG level during this $1 \mathrm{~s}$ ). The rmsEMG signals were displayed to the participants on a computer screen throughout the experiment so they can match the required levels of contraction during the tasks.

\subsection{Transcranial magnetic stimulation}

TMS was performed using a MagPro R30 (MagPro R30, MagVenture, Farum, DK) with single-pulse mode stimulator connected to a figure-of-eight coil with windings of $70 \mathrm{~mm}$ diameter. The TMS coil was positioned tangentially to the head over the M1 in order to induce a posterior-anterior current flow. The optimal sites of stimulation were determined by starting initial exploration over C3 and C4 standard landmarks of left and right M1, respectively (Jasper, 1958). Left and right M1 were determined as the stimulation site which elicited the largest and most consistent MEPs from the contralateral ECR muscle in at least 5 out of 10 trials. The optimal stimulation position "hot-spot" was marked on the scalp through the hair with ink to allow an accurate repositioning of the coil throughout the whole experiment. Resting motor threshold (RMT) was defined as the lowest TMS intensity eliciting MEPs of $50 \mu \mathrm{V}$ peak-to-peak in 5 out of 10 trials (Rossini et al., 1994). Active motor threshold 
(AMT) was defined as the lowest TMS intensity eliciting MEPs of $200 \mu \mathrm{V}$ peak-to-peak in 5 out of 10 trials (Rothwell et al., 1999). Corticospinal excitability was then determined for the left and right M1 at $130 \%$ of RMT and 130\% of AMT (Muthalib et al., 2017; Teo et al., 2015) for resting and active MEPs, respectively. The peak-topeak amplitude of these 12 evoked MEPs were normalized to the concomitant peripheral evoked response (see nerve stimulation section) to take account of possible alterations in signal conduction at any level from the motor cortex to within the muscle.

A $5 \mu \mathrm{V}$ pre-stimulus rmsEMG threshold was chosen to exclude subjects with a background EMG value too high that could potentially influence resting MEP amplitude. Since no subjects showed this effect, all $12 \mathrm{MEPs}$ were averaged and included for statistical analysis. Active MEPs and CSPs were evoked during wrist extension rmsEMG levels of $\sim 5 \%$ maximal rmsEMG (i.e., a-tDCS: left: $5.37 \pm 2.72 \%$, right: $6.9 \pm 3.49 \%$; LF-rTMS: left: $4.65 \pm 1.77 \%$, right: $6.58 \pm 2.95 \%$ ). CSP duration was defined as the interval between the onset of the MEP to the reappearance of at least $50 \%$ of the mean pre-TMS stimulus background EMG activity (Davidson \& Tremblay, 2013).

\subsection{Nerve stimulation}

The evoked M-wave potentials were obtained from both left and right ECR muscles by supramaximal electrical stimulation (DS7A, Digitimer, UK) of the radial nerve superior to the elbow joint. First, the position of the stimulation was determined by an initial exploration using a stylus. The stimulation site was determined as the site which elicits the largest and most consistent response for the minimal stimulation intensity. Afterward, the intensity of the stimulator was increased by $10 \mathrm{~mA}$ every $10 \mathrm{~s}$ until no further increase of the recorded M-wave amplitude was observed. The $\mathrm{M}_{\mathrm{MAX}}$ was defined as $130 \%$ of this intensity and was tested along with the TMS measurements at each time point.

\subsection{Repeated transcranial magnetic stimulation}

The rTMS was applied through the MagPro R30 biphasic magnetic generator on the right M1 representation of the non-dominant left ECR. The stimulation was delivered during 20 min at an intensity of $90 \%$ of RMT (Kaminski et al., 2013) with a $1 \mathrm{~Hz}$ frequency (1200 total pulses).

\subsection{Transcranial direct-current stimulation}

Direct electrical current was generated by a tDCS stimulator (Starstim ${ }^{\circledR}$, Neuroelectrics NE, Spain) and delivered to the left M1 representation of the dominant right ECR of the subject though a $4 \times 1$ ring $\mathrm{HD}$ a-tDCS montage with the active anode on the TMS "hot-spot" (see TMS measurement section) surrounded by four return electrodes, each at a distance of $35 \mathrm{~mm}$ from the active electrode (Muthalib, Besson, Rothwell, Ward, \& Perrey, 2016a). The five electrodes $\left(3.14 \mathrm{~cm}^{2} \mathrm{AgCl}\right.$ electrodes) were secured on the scalp using conductive paste $\left(\operatorname{Ten} 20^{\circledR}\right.$, Weaver and Company, USA). At the beginning of the sessions, stimulation ramped up during $30 \mathrm{~s}$ until the $2 \mathrm{~mA}$ current level was reached and continued at this level for a further 20 min duration.

\subsection{Statistical analysis}

Statistica 13.0 (Statsoft, Tulsa Oklahoma, USA) software was used for statistical analysis. Data were first screened for normality of distribution, homogeneity and sphericity of variances using a Shapiro-Wilk normality test, the Levene's and Mauchly's test, respectively A Greenhouse-Geisser correction was applied in case of sphericity violation in the analysis of variance (ANOVA). Following the ANOVA rules, post hoc LSD tests were performed only on significant main or interaction effects. All the Baseline TMS parameters, MEP, CSP and $\mathrm{M}_{\mathrm{MAX}}$ values were compared between sessions (LF-rTMS, aHDtDCS) by performing Wilcoxon tests.

A two-way repeated-measures (RM) ANOVAs were used on each M1 during each condition (Resting, Active) on the MEPs expressed as percent of $\mathrm{M}_{\mathrm{MAX}}\left(\mathrm{MEP} / \mathrm{M}_{\mathrm{MAX}}\right)$ and CSP values to assess the effects of the neuromodulation techniques. Partial eta-squared $\left(\mathrm{n}_{\mathrm{p}}{ }^{2}\right)$ values are reported as measures of effect size, with moderate and large effects considered for $\mathrm{n}_{\mathrm{p}}{ }^{2} \geq 0.06$ and $\mathrm{n}_{\mathrm{p}}{ }^{2} \geq 0.14$ respectively (Richardson, 2011). Results are present as mean \pm SD. Significant threshold was set at $p<0.05$.

\section{Results}

\subsection{Control measures}

No significant differences were found between aHDtDCS and LF-rTMS sessions for the Baseline TMS parameters (see Table 1), MEP, CSP and M-wave values. Also, no significant differences were found during and across sessions for the pre-stimulus rmsEMG to ensure no deleterious pre-activation effect over MEPs.

\subsection{CSP duration}

The two-way RM-ANOVA on the CSP values showed no influence $(144 \pm 30$ vs. $140 \pm 30$ vs. $135 \pm 20 \mathrm{~ms}$, from Baseline to Post-Stim to Post-Stim-20) of the neuromodulation $\left(\mathrm{F}_{2,16}=2.54 ; \mathrm{n}_{\mathrm{p}}{ }^{2}=0.24 ; p=0.11\right)$ with no significant difference $(140 \pm 30$ vs. $150 \pm 40 \mathrm{~ms}$ between tDCS and rTMS, respectively) between sessions $\left(\mathrm{F}_{1}\right.$, $\left.{ }_{8}=0.29 ; \mathrm{n}_{\mathrm{p}}{ }^{2}=0.04 ; p=0.6\right)$ or interaction $\left(\mathrm{F}_{2,16}=0.29\right.$; $\left.\mathrm{n}_{\mathrm{p}}{ }^{2}=0.04 ; p=0.75\right)$ in the left M1. Similarly, no neuromodulation influence $(143 \pm 20$ vs. $149 \pm 20$ vs. $147 \pm 20 \mathrm{~ms}$, from Baseline to Post-Stim to Post-Stim20) was found in the right $\mathrm{M} 1\left(\mathrm{~F}_{2,16}=0.31 ; \mathrm{n}_{\mathrm{p}}{ }^{2}=0.04\right.$; $p=0.74)$ with no significant difference $(142 \pm 20$ vs. $144 \pm 20 \mathrm{~ms}$ between tDCS and rTMS, respectively) between sessions $\left(\mathrm{F}_{1,8}=0.04 ; \mathrm{n}_{\mathrm{p}}{ }^{2}<0.01 ; p=0.85\right)$ or interaction $\left(\mathrm{F}_{2,16}=0.02 ; \mathrm{n}_{\mathrm{p}}{ }^{2}<0.01 ; p=0.98\right)$. 
Table 1. TMS parameters (expressed as \% of stimulator output) for each session. Resting (RMT) and active (AMT) motor thresholds were stable and not statistically different across sessions.

\begin{tabular}{lllll}
\hline & Left & Left & Right & Right \\
& M1-tDCS & M1-rTMS & M1-tDCS & M1-rTMS \\
\hline RMT & $45 \pm 6$ & $50 \pm 5$ & $46 \pm 4$ & $50 \pm 6$ \\
AMT & $40 \pm 6$ & $43 \pm 5$ & $43 \pm 5$ & $45 \pm 7$ \\
\hline
\end{tabular}

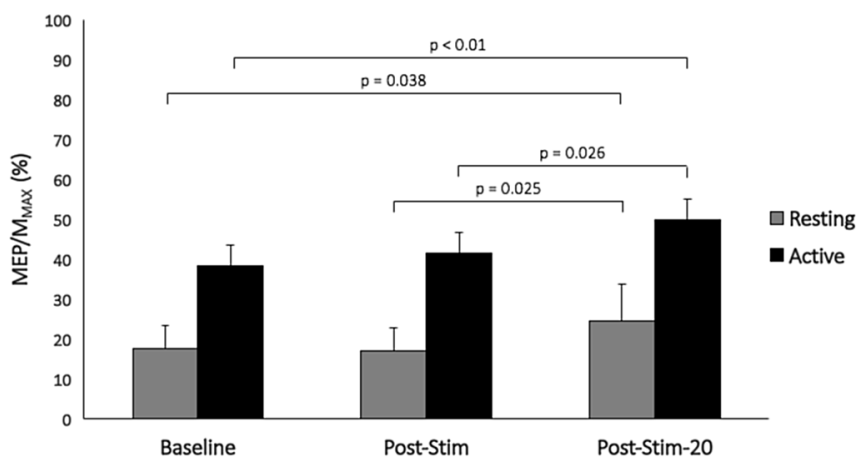

Figure 2. Excitability ( $\left.\mathrm{MEP} / \mathrm{M}_{\mathrm{MAx}}\right)$ changes in the right $\mathrm{M} 1$ immediately (Post-Stim) and $20 \mathrm{~min}$ (Post-Stim-20) following the neuromodulatory intervention (anodal high-density-tDCS and low frequency-repeated TMS) for resting and active MEPs. We show that both resting $(+38 \%)$ and active $(+30 \%)$ MEPs were significantly increased from Baseline to Post-Stim-20. Furthermore, both resting $(+44 \%)$ and active $(+20 \%)$ MEPs were significantly increased from Post-Stim to Post-Stim-20.

\subsection{MEP amplitude}

In the left M1, the two-way RM-ANOVA revealed no effect of the neuromodulatory intervention $\left(\mathrm{F}_{2,16}=0.24\right.$; $\left.\mathrm{n}_{\mathrm{p}}{ }^{2}=0.03 ; p=0.68\right)$ or any difference between sessions $\left(\mathrm{F}_{1,8}=0.78 ; \mathrm{n}_{\mathrm{p}}{ }^{2}=0.09 ; p=0.4\right)$ with no interaction $\left(\mathrm{F}_{2}\right.$, $\left.{ }_{16}=0.6 ; \mathrm{n}_{\mathrm{p}}{ }^{2}=0.07 ; p=0.48\right)$ for the resting MEPs. The active MEPs were also not influenced by the neuromodulation $\left(\mathrm{F}_{2,16}=0.12 ; \mathrm{n}_{\mathrm{p}}{ }^{2}=0.02 ; p=0.89\right)$ with any difference between sessions $\left(\mathrm{F}_{1}, 8=0.70 ; \mathrm{n}_{\mathrm{p}}{ }^{2}=0.08\right.$; $p=0.43)$ or any interaction $\left(\mathrm{F}_{2}, 16=1.28 ; \mathrm{n}_{\mathrm{p}}{ }^{2}=0.14\right.$; $p=0.3)$.

In the right $\mathrm{M} 1$, the RM-ANOVA revealed an effect of the neuromodulation $\left(\mathrm{F}_{2,16}=3.77 ; \mathrm{n}_{\mathrm{p}}{ }^{2}=0.32 ; p=0.045\right)$ without difference between sessions $\left(\mathrm{F}_{1,8}=0.1 ; \mathrm{n}_{\mathrm{p}}{ }^{2}=\right.$ $0.01 ; p=0.76)$ or interaction $\left(\mathrm{F}_{2,16}=1.21 ; \mathrm{n}_{\mathrm{p}}{ }^{2}=0.13\right.$; $p=0.32$ ) on the resting MEPs. Post hoc tests showed increased MEP values (see Figure 2) from Baseline to Post-Stim-20 $(+38 \% ; p=0.038)$ and from Post-Stim to Post-Stim-20 $(+44 \% ; p=0.025)$. Active MEPs were also influenced (see Figure 2) by the neuromodulation $\left(\mathrm{F}_{2}\right.$, $\left.{ }_{16}=6.14 ; \mathrm{n}_{\mathrm{p}}{ }^{2}=0.43 ; p=0.01\right)$ with no differences between sessions $\left(\mathrm{F}_{1,8}=0.21 ; \mathrm{n}_{\mathrm{p}}{ }^{2}=0.03 ; p=0.66\right)$ or interaction $\left(\mathrm{F}_{2,16}=1.07 ; \mathrm{n}_{\mathrm{p}}{ }^{2}=0.12 ; p=0.37\right)$. Post hoc tests revealed increased active MEPs values from Baseline to Post-Stim$20(+30 \% ; p<0.01)$ and from Post-Stim to Post-Stim-20 $(+20 \% ; p=0.026)$.

\section{Discussion}

In the present pilot study, we compared aHD-tDCS and LF-rTMS, two currently used neuromodulation techniques in the field, to enhance left M1 excitability. Against our hypothesis, we found that both aHD-tDCS of the left M1 and LF-rTMS of the right M1 did not significantly modulate left M1 excitability; rather we found a significantly increased right M1 (see Figure 2) excitability $20 \mathrm{~min}$ after the respective stimulation session. Thus, both aHD-tDCS and LF-rTMS sessions indiscriminately induced a facilitatory effect in the right M1 without modulating the left M1.

\subsection{Effects of LF-rTMS}

The overall increased right M1 excitability suggests that left M1 aHD-tDCS or right M1 LF-rTMS produced excitatory effects contrary to the expected inhibitory effects (Arai et al., 2007; Maeda et al., 2000). The reversal in the LF-rTMS effects is quite surprising but previous studies reported that $1 \mathrm{~Hz}$ LF-rTMS may not be inhibitory for everyone (see Figure 3) but excitatory in some subjects (Caparelli et al., 2012). This finding goes along with evidence on lack of real effects and the variability of the responses to rTMS (Hamada, Murase, Hasan, Balaratnam, \& Rothwell, 2013; Wassermann, 1998). For example, a review that analysed several rTMS studies reported that some of them displayed no effect following the rTMS protocol (Fitzgerald, Fountain, \& Daskalakis, 2006). Apart from variability in MEP responses, insufficient stimulation pulses may have also been a reason for LFrTMS effects. A study from Maeda et al. (2000) compared the MEP responses of subjects following 240 or 1600 pulses applied at frequencies of $1 \mathrm{~Hz}, 10 \mathrm{~Hz}, 15 \mathrm{~Hz}$ and $20 \mathrm{~Hz}$ rTMS at an intensity of $90 \%$ of RMT. They reported that $1 \mathrm{~Hz}$ rTMS at an intensity of $90 \%$ of RMT only significantly decreased MEP values in the stimulated M1 with 1600 pulses as compared to 240 pulses and added that interindividual variability was still high in the 1600 pulses condition with some subjects showing increased MEP amplitude. Therefore, an assumption is that our protocol consisting of 1200 pulses at $90 \%$ of RMT was likely still not sufficient to induce significant effects on MEP responses in all subjects.

The absence of decrease of CSP in the stimulated right M1 is providing another evidence that rTMS did not produce the expected inhibitory effects on the right M1. Interestingly, CSP was not modulated in the left M1, thus even though right M1 excitability was increased there was no effect on the opposite left M1 excitability and therefore no modulation of the interhemispheric interactions toward opposite left M1, contrary to previous studies (Gilio et al., 2003; Schambra et al., 2003). While it is in contradiction with the traditional interhemispheric balance model (Ferbert et al., 1992) it is in line with more recent critical point view on this model (Di Pino et al., 2014). Even with increased right M1 MEP changes, the absence of CSP modulation in the stimulated right M1 indicates the 


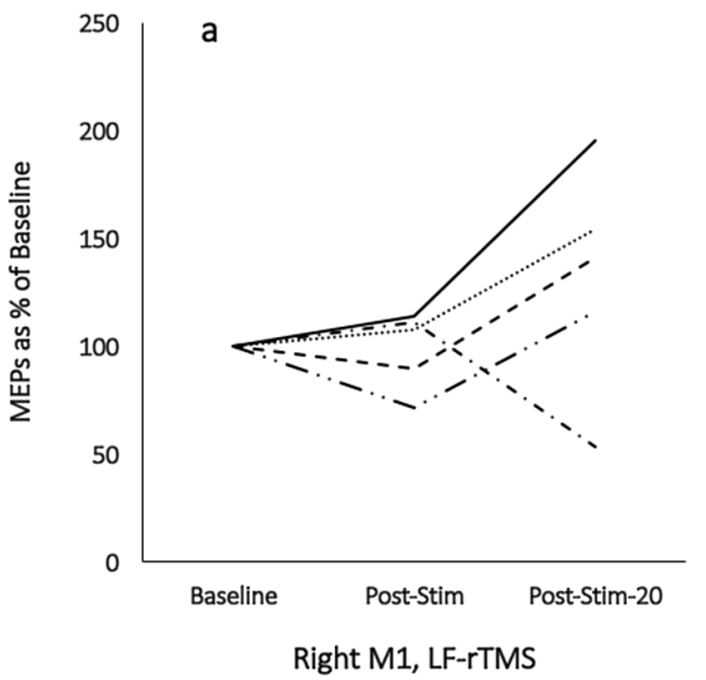

b

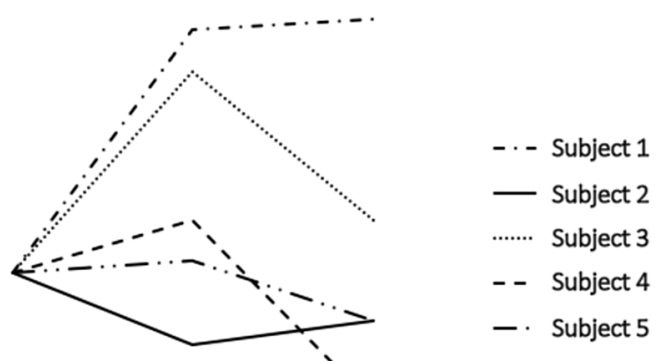

Baseline Post-Stim Post-Stim-20

Left M1, aHD-tDCS

Figure 3. Individual excitability (Post-Stim and Post-Stim-20 resting MEPs expressed as percent of baseline resting MEPs) changes in the right M1 following right M1 low frequency-repeated TMS (LF-rTMS; a, left panel) and in the left M1 following left M1 anodal high-density-tDCS (aHD-tDCS; b, right panel).

overall poor effect of LF-rTMS for modulating the inhibitory processes and thus may explain the absence of interhemispheric interaction modulation on the left M1.

\subsection{Effects of $t D C S$}

The left M1 aHD-tDCS intervention produced a surprising increase in excitability in the opposite right M1. Pioneer tDCS study claimed the impossibility to modulate the cortical excitability of the opposite M1 via transcallosal pathways (Lang, Nitsche, Paulus, Rothwell, \& Lemon, 2004). However, the increase of current intensities and protocol durations may bring this possibility as recent research reported contralateral effects of tDCS (Davidson, Bolic, \& Tremblay, 2016; Muthalib et al., 2016a; Tazoe, Endoh, Kitamura, \& Ogata, 2014; Teo et al., 2015). These studies show the direct impact of tDCS of one M1 on the opposite M1 most likely via transcallosal pathways.

The absence of effects of the tDCS on the stimulated left M1 is questionable. A well-known issue in the tDCS research field is the variability of MEP responses. Many studies demonstrated that subjects are responders and some subjects are non-responders (Wiethoff, Hamada, \& Rothwell, 2014) or even that subjects can switch from responders to non-responders between sessions (LopezAlonso, Fernandez-Del-Olmo, Costantini, Gonzalez-Henriquez, \& Cheeran, 2015) and vice versa. We thus assume that this variability along with the small sample played a role in our pilot study.

\subsection{Interhemispheric balance model}

Overall, the results tend to be contrary to the wellknown interhemispheric balance model often used to describe how brain hemispheres interact (Ferbert et al.,
1992; Meyer, Roricht, Grafin von Einsiedel, Kruggel, \& Weindl, 1995; Nowak et al., 2009). First, no modulation of excitability in the left M1 was observed and right M1 LFrTMS increased right M1 excitability. Furthermore, no decreased left M1 excitability was observed anyway. Also, left M1 aHD-tDCS did not modulate the stimulated left M1 but increased active right M1 MEP amplitude. However, these first results are quite inconclusive along with the small sample size. As reported by recent papers focusing on stroke (Chhatbar et al., 2016, 2017), modulating interhemispheric imbalance is possible. A hypothesis is that these studies performed in patients with interhemispheric excitability alterations allowed the neuromodulation to be more effective than in our healthy sample.

\section{Conclusion}

In this preliminary work, we have shown that neuromodulation techniques may not bring the expected effects. The low sample size may have been the primary reason to not being able to find the expected findings. However, the interesting finding of right M1 facilitatory effects with either right M1 LF-rTMS or left M1 aHD-tDCS provides some clue to changing preconceived ideas about models of how NIBS modulates local and interhemispheric pathways.

\section{Authors' contributions}

VC, JF and SP designed the study. VC conducted the study, data collection and data analysis. VC and SP prepared the manuscript draft with important intellectual input from JF and MM. All authors approved the final manuscript. 


\section{Glossary}

a-tDCS

anodal transcranial direct-current stimulation

AMT

CSP

ECR

EMG

aHD-tDCS

HF-rTMS high frequency repeated transcranial magnetic stimulation

LF-rTMS low-frequency repeated transcranial magnetic stimulation

M1 primary motor cortex

MEP motor-evoked potential

$\mathrm{M}_{\mathrm{MAX}} \quad$ maximal M-wave

NIBS

RMS

RMT

rTMS

tDCS

TMS

root mean square

resting motor threshold

repeated transcranial magnetic stimulation

transcranial direct-current stimulation

transcranial magnetic stimulation

Acknowledgment. Vincent Cabibel was supported by a Labex NUMEV Fellowship (Digital and Hardware Solutions, Environmental and Organic Life Modelling, ANR-10-LABX-20).

\section{References}

Arai, N., Okabe, S., Furubayashi, T., Mochizuki, H., Iwata, N. K., Hanajima, R., et al. (2007). Differences in after-effect between monophasic and biphasic high-frequency rTMS of the human motor cortex. Clinical Neurophysiology, 100/10, 59-67. DOI: 10.1016/j.clinph.2007.07.006.

Barker, A.T., Jalinoous, R., \& Freeston, I.L. (1985). Noninvasive magnetic stimulation of human motor cortex. The Lancet, 11/1, 1106-1107.

Caparelli, E.C., Backus, W., Telang, F., Wang, G.J., Maloney, T., Goldstein, R., \& Henn, F. (2012). Is $1 \mathrm{~Hz}$ rTMS Always Inhibitory in Healthy Individuals? The Open Neuroimaging Journal, 6, 69-74.

Chang, M.C., Kim, D.Y., \& Park, D.H. (2015). Enhancement of cortical excitability and lower limb motor function in patients with stroke by transcranial direct current stimulation. Brain Stimulation, 8/3, 561-566. DOI: 10.1016/j.brs.2015.01.411.

Chhatbar, P.Y., Ramakrishnan, V., Kautz, S., George, M.S., Adams, R.J., \& Feng, W. (2016). Transcranial direct current stimulation post-stroke upper extremity motor recovery studies exhibit a dose-response relationship. Brain Stimulation, 9/1, 16-26. DOI: 10.1016/j.brs.2015.09.002.

Chhatbar, P.Y., Chen, R., Deardorff, R., Dellenbach, B., Kautz, S.A., George, M.S., \& Feng, W. (2017). Safety and tolerability of transcranial direct current stimulation to stroke patients A phase I current escalation study. Brain Stimulation. DOI: 10.1016/j.brs.2017.02.007.

Corti, M., Patten, C., \& Triggs, W. (2012). Repetitivetranscranial magnetic stimulation of motor cortex after stroke: a focused review. American Journal of Physical Medicine \& Rehabilitation, 91/3, 254-270. DOI: 10.1097/PHM.0b013e318228bf0c.

Davidson, T., \& Tremblay, F. (2013). Hemispheric differences in corticospinal excitability and in transcallosal inhibition in relation to degree of handedness. PLoS One, 8/7, e70286. DOI: 10.1371/journal.pone.0070286.

Davidson, T.W., Bolic, M., \& Tremblay, F. (2016). Predicting modulation in corticomotor excitability and in transcallosal inhibition in response to anodal transcranial direct current stimulation. Frontiers in Human Neuroscience, 10, 49. DOI: 10.3389/fnhum.2016.00049.

Di Lazzaro, V., Dileone, M., Capone, F., Pellegrino, G., Ranieri, F., Musumeci, G., et al. (2014). Immediate and late modulation of interhemipheric imbalance with bilateral transcranial direct current stimulation in acute stroke. Brain Stimulation, 7/6, 841-848. DOI: 10.1016/j.brs.2014.10.001.

Di Pino, G., Pellegrino, G., Assenza, G., Capone, F., Ferreri, F., Formica, D., et al. (2014). Modulation of brain plasticity in stroke: a novel model for neurorehabilitation. Nature Reviews Neurology, 10/10, 597-608. DOI: 10.1038/nrneurol.2014.162.

Dobek, C.E., Blumberger, D.M., Downar, J., Daskalakis, Z.J., \& Vila-Rodriguez, F. (2015). Risk of seizures in transcranial magnetic stimulation: a clinical review to inform consent process focused on bupropion. Neuropsychiatric Disease and Treatment, 11, 2975-2987. DOI: 10.2147/NDT. S91126Risk.10.2147/NDT.S91126Neuropsychiatric.

Edwards, D., Cortes, M., Datta, A., Minhas, P., Wassermann, E. M., \& Bikson, M. (2013). Physiological and modeling evidence for focal transcranial electrical brain stimulation in humans: a basis for high-definition tDCS. Neuroimage, 74 , 266-275. DOI: 10.1016/j.neuroimage.2013.01.042.

Ferbert, A., Priori, A., Rothwell, J.C., Day, B.L., Colebatch, J. G., \& Mardsen, C.D. (1992). Interhemispheric inhibition of the human motor cortex. Journal of Physiology, 453, 525-546.

Fitzgerald, P.B., Fountain, S., \& Daskalakis, Z.J. (2006). A comprehensive review of the effects of rTMS on motor cortical excitability and inhibition. Clinical Neurophysiology, 117/12, 2584-2596. DOI: 10.1016/j.clinph.2006.06.712.

Gandiga, P.C., Hummel, F.C., \& Cohen, L.G. (2006). Transcranial DC stimulation (tDCS): a tool for double-blind sham-controlled clinical studies in brain stimulation. Clinical Neurophysiology, 117/4, 845-850. DOI: 10.1016/j. clinph.2005.12.003.

Gilio, F., Rizzo, V., Siebner, H.R., \& Rothwell, J.C. (2003). Effects on the right motor hand-area excitability produced by low-frequency rTMS over human contralateral homologous cortex. The Journal of Physiology, 551/Pt 2, 563-573. DOI: 10.1113/jphysiol.2003.044313.

Hamada, M., Murase, N., Hasan, A., Balaratnam, M., \& Rothwell, J.C. (2013). The role of interneuron networks in driving human motor cortical plasticity. Cerebral Cortex, 23/ 7. 1593-1605. DOI: 10.1093/cercor/bhs147.

Hao, Z., Wang, D., Zeng, Y., \& Liu, M. (2013). Repetitive transcranial magnetic stimulation for improving function after stroke (Review). Cochrane Database of Systematic Revieus, 5, CD008862.

Hendy, A.M., \& Kidgell, D.J. (2014). Anodal-tDCS applied during unilateral strength training increases strength and corticospinal excitability in the untrained homologous muscle. Experimental Brain Research, 232/10, 3243-3252. DOI: $10.1007 / \mathrm{s} 00221-014-4016-8$.

Hermens, H.J., Freriks, B., Disselhorst-Klug, C., \& Rau, G. (2000). The recommendations for sensors and sensor placement procedures for surface electromyography. Journal of Electromyography and Kinesiology, 10/5, 361-374.

Hoyer, E.H., \& Celnik, P.A. (2011). Understanding and enhancing motor recovery after stroke using transcranial magnetic stimulation. Restorative Neurology and Neuroscience, 29/6, 395-409. DOI: 10.3233/RNN-2011-0611. 
Jasper, H.H. (1958). Report of the committee on methods of clinical examination in electroencephalography. Electroencephalography and Clinical Neurophysiology, 10, 370-375.

Jeffery, D.T., Norton, J.A., Roy, F.D., \& Gorassini, M.A. (2007). Effects of transcranial direct current stimulation on the excitability of the leg motor cortex. Experimental Brain Research, 182/2, 281-287. DOI: 10.1007/s00221007-1093-y.

Jo, J.M., Kim, Y.H., Ko, M.H., Ohn, S.H., Joen, B., \& Lee, K.H. (2009). Enhancing the working memory of stroke patients using tDCS. American Journal of Physical Medicine $\&$ Rehabilitation, 88/5, 404-409. DOI: 10.1097/PHM.0b013e3181a0e4cb.

Kaminski, E., Hoff, M., Sehm, B., Taubert, M., Conde, V., Steele, C.J., et al. (2013). Effect of transcranial direct current stimulation (tDCS) during complex whole body motor skill learning. Neuroscience Letters, 552, 76-80. DOI: 10.1016/j. neulet.2013.07.034.

Kuo, H.I., Bikson, M., Datta, A., Minhas, P., Paulus, W., Kuo, M.F., \& Nitsche, M.A. (2013). Comparing cortical plasticity induced by conventional and high-definition $4 \times 1$ ring tDCS: a neurophysiological study. Brain Stimulation, 6/4, 644-648. DOI: $10.1016 /$ j.brs.2012.09.010.

Lang, N., Nitsche, M.A., Paulus, W., Rothwell, J.C., \& Lemon, R.N. (2004). Effects of transcranial direct current stimulation over the human motor cortex on corticospinal and transcallosal excitability. Experimental Brain Research, 156/4, 439-443. DOI: 10.1007/s00221-003-1800-2.

Li, H., Zhao, G., Zhou, Y., Chen, X., Ji, Z., \& Wang, L. (2014). Relationship of EMG/SMG features and muscle strength level: an exploratory study on tibialis anterior muscles during plantar-flexion among hemiplegia patients. Biomedical Engineering Online, 13, 5. DOI: 10.1186/1475925X-13-5.

Lopez-Alonso, V., Fernandez-Del-Olmo, M., Costantini, A., Gonzalez-Henriquez, J.J., \& Cheeran, B. (2015). Intraindividual variability in the response to anodal transcranial direct current stimulation. Clinical Neurophysiology, 126/12, 2342-2347. DOI: 10.1016/j.clinph.2015.03.022.

Maeda, F., Keenan, J.P., Tormos, J.M., Topka, H., \& PascualLeone, A. (2000). Interindividual variability of the modulatory effects of repetitive transcranial magnetic stimulation on cortical excitability. Experimental Brain Research, 133/4, 425-430. DOI: $10.1007 / \mathrm{s} 002210000432$.

Meyer, B.U., Roricht, S., Grafin von Einsiedel, H., Kruggel, F., \& Weindl, A. (1995). Inhibitory and excitatory interhemispheric transfers between motor cortical areas in normal humans and patients with abnormalities of the corpus callosum. Brain, 118, 429-440.

Monte-Silva, K., Kuo, M.F., Hessenthaler, S., Fresnoza, S., Liebetanz, D., Paulus, W., \& Nitsche, M.A. (2013). Induction of late LTP-like plasticity in the human motor cortex by repeated non-invasive brain stimulation. Brain Stimulation, 6/3, 424-432. DOI: 10.1016/j.brs.2012.04.011.

Muthalib, M., Besson, P., Rothwell, J.C., Ward, E.T., \& Perrey, S. (2016a). Effects of anodal high-definition transcranial direct current stimlation on bilateral sensorimotor cortex activation during sequential finger movements: an fNIRS study. Advances in Experimental Medicine and Biology, 876, 351-359.

Muthalib, M., Besson, P., Rothwell, J.C., Ward, T., \& Perrey, S. (2016b). Effects of anodal high-definition transcranial direct current stimulation on bilateral sensorimotor cortex activation during sequential finger movements: an fNIRS study. Advances in Experimental Medicine and Biology, 876, 351359. DOI: 10.1007/978-1-4939-3023-4_44.
Muthalib, M., Cabibel, V., Rothwell, J.C., Teo, W.P., \& Perrey, S. (2017). Effects of HD-tDCS and crossed-facilitation on interhemispheric interactions. Brain Stimulation, 10, 346540. DOI: $10.1016 /$ j.brs.2017.01.356.

Nitsche, M.A., \& Paulus, W. (2000). Excitability changes induced in the human motor cortex by weak transcranial direct current stimulation. Journal of Physiology, 527/3, 633-639.

Nowak, D.A., Grefkes, C., Ameli, M., \& Fink, G.R. (2009). Interhemispheric competition after stroke" brain stimulation to enhance recovery of function of the affected hand. Neurorehabilitation and Neural Repair, 23/7, 641-656.

Oldfield, R.C. (1971). The assessment and analysis of handedness: the Edinburgh inventory. Neuropsychologia, 9, 97-113.

Pereira, L.S., Muller, V.T., da Mota Gomes, M., Rotenberg, A., \& Fregni, F. (2016). Safety of repetitive transcranial magnetic stimulation in patients with epilepsy: A systematic review. Epilepsy $\&$ behavior, 57/Pt A, 167-176. DOI: 10.1016/j. yebeh.2016.01.015.

Priori, A., Hallett, M., \& Rothwell, J.C. (2009). Repetitive transcranial magnetic stimulation or transcranial direct current stimulation? Brain Stimulation, 2/4, 241-245. DOI: 10.1016/j.brs.2009.02.004.

Richardson, J.T.E. (2011). Eta squared and partial eta squared as measures of effect size in educational research. Educational Research Review, 6/2, 135-147. DOI: 10.1016/j. edurev.2010.12.001.

Ridding, M.C., \& Rothwell, J.C. (2007). Is there a future for therapeutic use of transcranial magnetic stimulation? Nature Neuroscience, 8, 559-568.

Rossini, P.M., Barker, A.T., Berardelli, A., Caramia, M.D., Caruso, G., Cracco, R.Q., et al. (1994). Non-invasive electrical and magnetic stimulation of the brain, spinal cord and roots: basic principles and procedures fort routine clinical application. Report of an IFCN committee. Electroencephalography and Clinical Neurophysiology, 91, 79-92.

Rothwell, J.C., Hallett, M., Berardelli, A., Eisen, A., Rossini, P. M., \& Paulus, W. (1999). Magnetic stimulation: motor evoked potentials. Electroencephalography and Clinical Neurophysiology, 52, 97-103.

Schambra, H.M., Sawaki, L., \& Cohen, L.G. (2003). Modulation of excitability of human motor cortex (M1) by $1 \mathrm{~Hz}$ transcranial magnetic stimulation of the contralateral M1. Clinical Neurophysiology, 114, 130-133.

Schlaug, G., Renga, V., \& Nair, D. (2008). Transcranial direct current stimulation in stroke recovery. Archives of Neurology, 65/12, 1571-1576. DOI: 10.1001/archneur.65.12.1571.

Schulz, R., Gerloff, C., \& Hummel, F.C. (2013). Non-invasive brain stimulation in neurological diseases. Neuropharmacology, 64, 579-587. DOI: 10.1016/j.neuropharm.2012.05.016.

Simis, M., Adeyemo, B.O., Medeiros, L.F., Miraval, F., Gagliardi, R.J., \& Fregni, F. (2013). Motor cortex-induced plasticity by noninvasive brain stimulation: a comparison between transcranial direct current stimulation and transcranial magnetic stimulation. Neuroreport, 24/17, 973-975. DOI: $10.1097 /$ WNR.0000000000000021.

Simonetta-Moreau, M. (2014). Non-invasive brain stimulation (NIBS) and motor recovery after stroke. Annals of Physical and Rehabilitation Medicine, 57/8, 530-542. DOI: 10.1016/j. rehab.2014.08.003.

Tazoe, T., Endoh, T., Kitamura, T., \& Ogata, T. (2014). Polarity specific effects of transcranial direct current stimulation on interhemispheric inhibition. PLoS One, 9/ 12, e114244. DOI: 10.1371/journal.pone.0114244. 
Teo, W.P., Muthalib, M., Kidgell, D., Frazer, A., Hendy, A., Goodwill, A.M., \& Perrey, S. (2015). Ipsilateral M1 transcranial direct current stimulation increases excitability of the contralateral M1 during an active motor task: Implications for stroke rehabilitation. Annals of Physical and Rehabilitation Medicine, 58, e1-e2.

Villamar, M.F., Volz, M.S., Bikson, M., Datta, A., Dasilva, A.F., \& Fregni, F. (2013). Technique and considerations in the use of $4 \times 1$ ring high-definition transcranial direct current stimulation (HD-tDCS). Journal of Visualized Experiments, 77, e50309. DOI: 10.3791/50309.

Wassermann, E.M. (1998). Risk and safety of repetitive transcranial magnetic stimulation: report and suggested guidelines. Electroencephalography and Clinical Neurophysiology, 108, 1-16.

Wiethoff, S., Hamada, M., \& Rothwell, J.C. (2014). Variability in response to transcranial direct current stimulation of the motor cortex. Brain Stimulation, 7/3, 468-475. DOI: 10.1016/ j.brs.2014.02.003.

Cite this article as: Cabibel V, Muthalib M, Froger J, \& Perrey S (2018) Comparison of repeated transcranial stimulation and transcranial direct-current stimulation on primary motor cortex excitability and inhibition: A pilot study. Mov Sport Sci/Sci Mot, 100, 59-67 